\title{
OPEN Impact of noise on development, physiological stress and behavioural patterns in larval zebrafish
}

\begin{abstract}
Rafael A. Lara ${ }^{1,2 \bowtie}$ \& Raquel O. Vasconcelos ${ }^{1 \bowtie}$
Noise pollution is increasingly present in aquatic ecosystems, causing detrimental effects on growth, physiology and behaviour of organisms. However, limited information exists on how this stressor affects animals in early ontogeny, a critical period for development and establishment of phenotypic traits. We tested the effects of chronic noise exposure to increasing levels (130 and $150 \mathrm{~dB}$ re $1 \mu \mathrm{Pa}$, continuous white noise) and different temporal regimes on larval zebrafish (Danio rerio), an important vertebrate model in ecotoxicology. The acoustic treatments did not affect general development or hatching but higher noise levels led to increased mortality. The cardiac rate, yolk sac consumption and cortisol levels increased significantly with increasing noise level at both 3 and 5 dpf (days post fertilization). Variation in noise temporal patterns (different random noise periods to simulate shipping activity) suggested that the time regime is more important than the total duration of noise exposure to down-regulate physiological stress. Moreover, $5 \mathrm{dpf}$ larvae exposed to $150 \mathrm{~dB}$ continuous noise displayed increased dark avoidance in anxiety-related dark/light preference test and impaired spontaneous alternation behaviour. We provide first evidence of noise-induced physiological stress and behavioural disturbance in larval zebrafish, showing that both noise amplitude and timing negatively impact key developmental endpoints in early ontogeny.
\end{abstract}

Anthropogenic noise has increased unprecedentedly in the last century both on land and underwater, being considered a global environmental pollutant by international legislation ${ }^{1-3}$. Noise pollution derives mostly from traffic, industry, resource extraction, construction and recreational activities, and it is expanding in time and space with subsequent negative impacts on human health and wildlife ${ }^{4-6}$. Besides a significant increase in overall sound levels, anthropogenic noise sources add new sounds into the environment that differ greatly in spectral composition and duty cycle from the natural soundscapes ${ }^{7,8}$. For instance, shipping and recreational boats add a broadband noise component into the overall aquatic acoustic scene that lasts much longer compared to pile driving and seismic air guns that generate impulsive short-lasting low frequency sounds $\mathrm{s}^{9,10}$.

Repeated or chronic exposure to increased noise levels can affect how animals respond to this stressor due to mechanisms of habituation and sensitization that rely on either augmented or decreased tolerance, respectively ${ }^{11-15}$. Shifts in tolerance to noise exposure, or to any other stressor, typically depend on duration, intensity and time regime of exposure ${ }^{11,16}$, hence identifying patterns with less impact is paramount for defining sustainable noise management and mitigation strategies. Noise is known to cause a myriad of detrimental effects in various taxa including auditory impairment ${ }^{17-20}$, impaired development ${ }^{21,22}$, heightened physiological stress $^{8,19,23,24}$ and behavioural disturbance ${ }^{7,19,20,23,25}$, thus posing unprecedented risks on species survival, biodiversity and ultimately on ecosystems health.

Considering how fast aquatic soundscapes are changing ${ }^{26}$, it is fundamental to develop research on how different noise regimes affect development, physiology and behaviour of fish, as they are key components of most aquatic ecosystem ${ }^{27}$. An increasing amount of studies are showing the negative effects of anthropogenic noise on fish species, including lowered survivability ${ }^{28}$, impaired growth ${ }^{13,29}$, increased physiological stress ${ }^{30,31}$, compromised hearing ${ }^{32,33}$, and behavioural disturbance ${ }^{34,35}$. The majority of these studies, however, focused on the effects of short-term exposure to intense noise amplitudes, and most of the impact is likely to derive from less noticeable mild noise levels and repeated/chronic exposure ${ }^{36}$. This type of exposure can introduce modifications

${ }^{1}$ Institute of Science and Environment, University of Saint Joseph, Macao S.A.R., China. ${ }^{2}$ Departamento de Biología, Universidad de Sevilla, Seville, Spain. ${ }^{\circledR}$ email: rafael.lara@usj.edu.mo; raquel.vasconcelos@usj.edu.mo 
on how species respond to the stressor due to changes across time and cumulative effects, as well as impose driving evolutionary pressures ${ }^{27,37,38}$.

Only few studies have evaluated long-term noise effects on animals ${ }^{19,20,39,40}$ and particularly in early ontogeny ${ }^{41,42}$, a critical period for development and establishment of phenotypic traits ${ }^{43}$. In fish, very scarce information exists on how chronic exposure to this environmental stressor impacts species in the adult stage ${ }^{31,44,45}$ and the effects on larval survivability ${ }^{46}$, development and behaviour ${ }^{13,47}$, and physiological stress ${ }^{48}$. So far, only one study evaluated how prolonged noise exposure varying in regularity can impact fish in early development ${ }^{13}$. This study demonstrated that two days of either regular or random noise may affect growth, while regular regime also led to faster yolk sac consumption and altered body condition in larval Atlantic cod Gadus morhua. Further research is needed regarding the impact of chronic noise exposure on early development using model organisms that allow for integrative studies combining morphological, physiological, behavioural and genetic approaches, in order to unravel coping mechanisms of acoustic stress.

The zebrafish Danio rerio has become a reference vertebrate model in the fields of developmental biology ${ }^{49}$, ecotoxicology $y^{50,51}$ and hearing research ${ }^{52}$. This species has been further used to test the impact of noise exposure on lateral line hair cells ${ }^{53}$ and auditory-evoked escape responses ${ }^{54}$, but the effects of acoustic stress on development, physiology and anxiety-related behaviors remain unknown.

In the present study we performed a split-brood experiment to test the effects of chronic exposure to noise of different amplitude levels and temporal patterns (random intermittent regimes simulating shipping activity) on development, physiological stress and behavioural traits in larval zebrafish. We used ecologically-relevant mild noise levels, such as 130 and $150 \mathrm{~dB}$ re $1 \mu \mathrm{Pa}$, which are representative of boat noise and amplitudes found in zebrafish housing systems ${ }^{55}$.

\section{Results}

Hatching, growth and mortality. The noise treatments used in this study did not significantly affect the hatching rate of zebrafish embryos (comparison between noise levels-control, $130 \mathrm{~dB}\left(\mathrm{CN}_{130}\right)$ and $150 \mathrm{~dB}\left(\mathrm{CN}_{150}\right): \mathrm{F}_{(2,38)}=2.80, p=0.074$; and temporal variations-control, $\mathrm{CN}, \mathrm{IN} 1, \mathrm{IN} 2, \mathrm{IN} 3: \mathrm{F}_{(4,47)}=0.76$, $p=0.25$ ), nor cause any obvious developmental abnormalities. Embryos started hatching at 2 dpf (i.e. control: $10-11 \%$; $\mathrm{CN}_{130}: 9-10 \% ; \mathrm{CN}_{150}: 7-8 \%$, without differences between treatments and by $3 \mathrm{dpf}$ all viable specimens hatched. Furthermore, changes in both noise level and temporal patterns did not affect the total length of the larval zebrafish measured at $3 \mathrm{dpf}$ (noise levels: $\mathrm{F}_{(2,131)}=0.32, p=0.655$; temporal variation: $\mathrm{F}_{(4,147)}=5.19, p=0.66$ ) nor at $5 \mathrm{dpf}$ (noise levels: $\mathrm{F}_{(2,131)}=0.17, p=0.735$; temporal variation: $\mathrm{F}_{(4,143)}=0.64, p=0.83$ ).

However, an effect of noise exposure on mortality was observed throughout the acoustic treatments for specimens under continuous noise $(\mathrm{CN})\left(\mathrm{F}_{(2,38)}=8.71, p<0.001\right)$, with $\mathrm{CN}_{150}$ causing a significant increase compared to $\mathrm{CN}_{130}(p=0.036)$ and control $(p<0.001)$ (Fig. 1A,C). Variation in the timing of acoustic disturbances also induced an increase in mortality in all the noise exposed groups compared to control $\left(\mathrm{F}_{(4,47)}=3.78, p=0.005\right)$ (Fig. 1B,D). Short (IN1) and medium (IN2) noise periods of intermittent treatments induced higher mortality rates similar to $\mathrm{CN}$ (IN1 vs. control: $p=0.008$; IN2 vs. control: $p=0.015$ ), while the treatment presenting long noise segments (IN3) did not induce significant mortality compared to control ( $p=0.066)$ (Fig. 1B,D).

Physiological stress indicators. Cardiac rate, yolk sac consumption and cortisol levels increased significantly with increasing noise level, which clearly indicated higher physiological stress. The cardiac rate of larval zebrafish at $3 \mathrm{dpf}$ was about $173 \pm 30 \mathrm{bpm}$ (mean \pm SEM, standard error of the mean; bpm, beats per minute) for control and increased to $191 \pm 60 \mathrm{bpm}$ after playback of $\mathrm{CN}_{150}$, whilst at $5 \mathrm{dpf}$ it was around $203 \pm 40$ (control) and increased to $224 \pm 50 \mathrm{bpm}$ after $\mathrm{CN}_{150}$ treatment. A significant increase was verified with increasing noise level in both $3 \mathrm{dpf}\left(\mathrm{F}_{(2,134)}=4.20, p=0.004\right)$ and $5 \mathrm{dpf}$ larvae $\left(\mathrm{F}_{(2,133)}=7.17, p=0.002\right)$, with $\mathrm{CN}_{150}$ causing the highest differences compared to control $(p<0.001)$ (Fig. $2 \mathrm{~A})$.

Similarly, the yolk sac consumption increased significantly with increasing noise level at both $3 \mathrm{dpf}$ $\left(\mathrm{F}_{(2,136)}=11.96, p<0.001\right)$ and $5 \mathrm{dpf}$ larvae $\left(\mathrm{F}_{(2,134)}=16.59, p<0.001\right)$, with $\mathrm{CN}_{150}$ also inducing the highest differences compared to control $(p<0.001)$ (Fig. $2 \mathrm{~B})$.

Variation in the timing of the acoustic disturbances caused different effects on both cardiac rate and yolk sac consumption, suggesting that noise temporal regime is important to regulate physiological stress and depletion of embryonic endogenous energy reserves. The cardiac rate was significantly affected by noise time variations at both $3 \mathrm{dpf}\left(\mathrm{F}_{(4,146)}=25.36, p<0.001\right)$ and $5 \mathrm{dpf}\left(\mathrm{F}_{(4,143)}=15.50, p<0.001\right)$, with continuous noise causing the highest impact compared to intermittent treatments at $5 \mathrm{dpf}(p<0.001)$ (Fig. 2C). IN3 consistently induced the lowest impact among intermittent regimes at both 3 and $5 \mathrm{dpf}$ compared to control $(p=0.009$ and $p=0.046$, respectively). Additionally, significant differences in the yolk sac consumption were found between these noise treatments at $3 \mathrm{dpf}\left(\mathrm{F}_{(4,145)}=13.79, p<0.001\right)$ and $5 \mathrm{dpf}\left(\mathrm{F}_{(4,143)}=12.19, p<0.001\right)$ (Fig. $\left.2 \mathrm{D}\right)$. CN induced the highest yolk sac consumption compared to intermittent noise groups at $5 \mathrm{dpf}(p<0.001)$.

Overall, the cardiac rate was negatively correlated with yolk sac size $(\mathrm{R}=-0.61, \mathrm{~N}=370, p<0.001)$, meaning that individuals with higher cardiac rate consumed their energy reserves faster (Fig. 2E).

Noise-induced physiological stress was confirmed through whole-body cortisol quantification. Cortisol levels increased significantly with noise amplitude at both $3 \mathrm{dpf}\left(\mathrm{F}_{(2,35)}=4.84, p=0.014 ; \mathrm{CN}_{150}\right.$ higher than control $p=0.010)$ and $5 \mathrm{dpf}\left(\mathrm{F}_{(2,29)}=4.37 ; p=0.023\right.$; both $\mathrm{CN}_{130}$ and $\mathrm{CN}_{150}$ higher than control $p=0.016$ and $p=0.004$ respectively) (Fig. $3 \mathrm{~A}$ ). Variation in the noise time regime caused changes in cortisol, however, they were not statistically significant $-3 \mathrm{dpf}\left(\mathrm{F}_{(4,45)}=2.23, p=0.082\right) ; 5 \mathrm{dpf}\left(\mathrm{F}_{(4,35)}=2.65, p=0.052\right)$ (Fig. 3B).

Behavioural patterns. In order to assess potential changes at the behavioural level, $5 \mathrm{dpf}$ larvae exposed to $150 \mathrm{~dB}$ continuous noise $\left(\mathrm{CN}_{150}\right)$ were further tested using an anxiety-related light/dark preference test (Fig. 4A). 


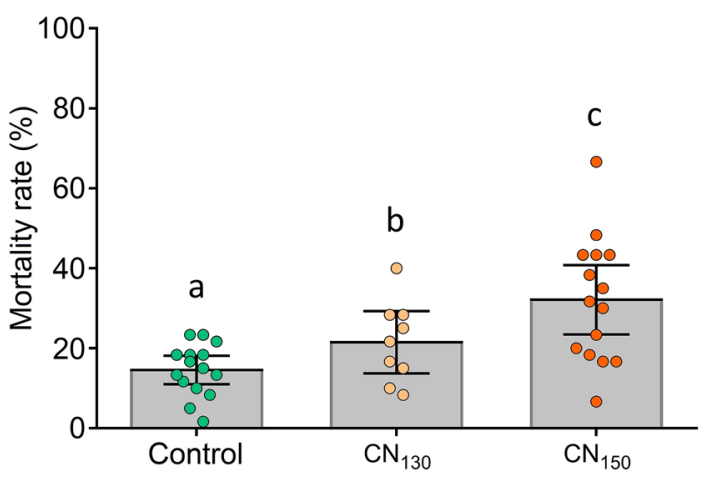

A
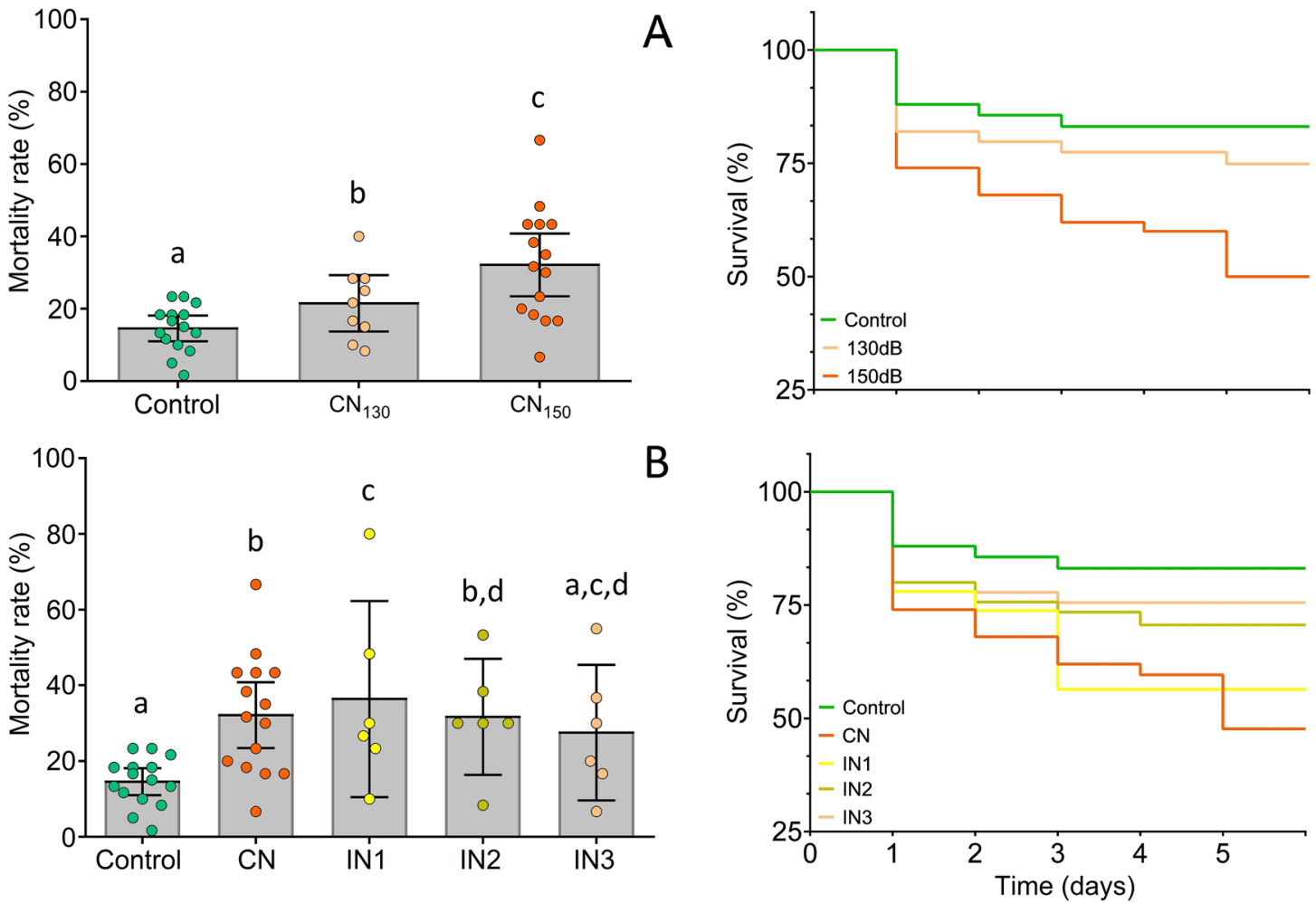

Figure 1. Comparison of mean mortality rate between treatment groups (larval zebrafish up to 5 days post fertilization) exposed to $(\mathbf{A})$ continuous noise at different amplitudes $\left(\mathrm{F}_{(2,38)}=8.71, p<0.001\right)$ and $(\mathbf{B})$ varying noise temporal patterns $\left(\mathrm{F}_{(4,47)}=3.78, p=0.005\right)$. Kaplan-Meier percentual survival plot of zebrafish larvae throughout the 5-days of chronic acoustic treatments of $(\mathbf{C})$ increasing noise amplitudes and (D) varying temporal patterns. Control- silent conditions, $\mathrm{CN}$ - continous noise at either $130\left(\mathrm{CN}_{130}\right)$ or $150 \mathrm{~dB}$ re $1 \mu \mathrm{Pa}$ $\left(\mathrm{CN}_{150}\right)$, IN- intermittent regime with short (IN1), medium (IN2) and long noise segments (IN3). Error bars represent $95 \%$ confidence intervals. Different letters indicate statistically significant differences between specific groups based on post hoc tests.

Specimens exposed to the acoustic stressor exhibited stronger dark avoidance or scotophobia, as measured based on a choice index $\left(\mathrm{U}_{(2,173)}=5341.50 ; p<0.001\right)$ (Fig. 4B).

Larval zebrafish ( $5 \mathrm{dpf})$ were also tested regarding exploratory behaviour using the Spontaneous Alternation Behaviour (SAB) assay (Fig. 5A), which describes the tendency of animals to alternate their turn direction in consecutive turns ${ }^{56,57}$. The $\mathrm{SAB}$ assay revealed that $70 \%$ of larvae reared under silent control conditions exhibited normal swimming alternation, as opposed to only $34 \%$ from the noise treatment group $\left(\mathrm{CN}_{150}\right)\left(\mathrm{t}_{(113)}=-4.08\right.$, $p<0.001$ ) (Fig. 5B). The swimming patterns of these specimens were further investigated in an open field arena, which revealed a reduction in covered area for the noise-treated larvae $\left(\mathrm{t}_{(89)}=7.33, p<0.001\right)$.

\section{Discussion}

To our knowledge this is the first study assessing the impact of noise exposure on larval zebrafish (Danio rerio), a reference model organism in ecotoxicology. We provide evidence of noise-induced physiological and behavioural disturbance in larval zebrafish, and demonstrate that both amplitude and timing of the acoustic stressor may impact key health-related endpoints in early ontogeny.

The acoustic treatments considered in the present study did not affect hatching or general development but higher noise levels ( 130 and $150 \mathrm{~dB}$ re $1 \mu \mathrm{Pa}$ ) led to increased mortality (from $15 \%$ in control up to $33 \%$ at the highest sound level). Very limited information exists on the impact of noise on fish hatching success and survival. Banner and Hyatt ${ }^{46}$ found significant lethal effects on fish embryos (Cyprinodon variegatus, Cyprinodontidae) exposed to higher noise conditions in a tank system with two distinct acoustic zones, but no effect was detected at the post hatching stage ( $>24 \mathrm{hpf}$ ). Bruintjes and Radford ${ }^{58}$ evaluated the impact of small motorboat noise (127 dB re $1 \mu \mathrm{Pa}$ RMS) on a cichlid fish (Neolamprologus pulcher) and did not find an effect on hatching success or fry survival.

Previous studies described mixed results in regards to the impact of noise on fish growth. Banner and Hyatt ${ }^{46}$ reported noise-induced decrease in larval growth during the first 11-15 days post-hatch, whereas Bruintjes and Radford ${ }^{58}$ described an absence of effects on body length or weight after 4 weeks post-hatch. Both Davidson et al. ${ }^{59}$ and Nedelec et al. ${ }^{13}$ found that increased noise levels significantly reduced development in different fish species (rainbow trout Oncorhynchus mykiss and Atlantic cod Gadus morhua), however this was followed by a catch-up growth at later stages. 

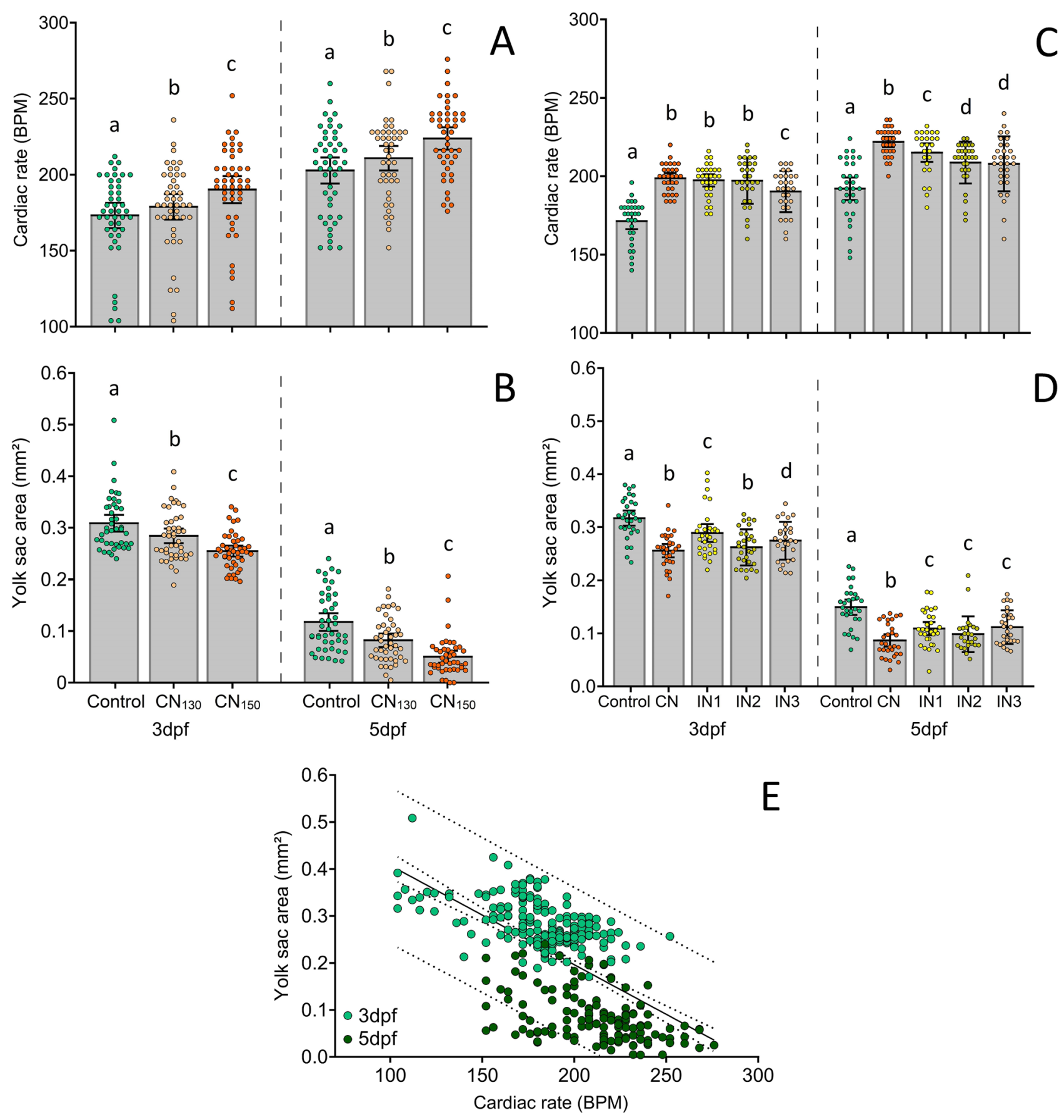

Figure 2. Comparison of mean cardiac rate and yolk sac area of larval zebrafish with 3 and 5 dpf (days post fertilization) exposed to different noise amplitudes (A and $\mathbf{B})$ and temporal patterns at $150 \mathrm{~dB}$ re $1 \mu \mathrm{Pa}(\mathbf{C}$ and D). Increasing noise amplitudes induced heightened cardiac rate at $3 \mathrm{dpf}\left(\mathrm{F}_{(2,134)}=4.20, p=0.004\right)$ and $5 \mathrm{dpf}$ $\left(\mathrm{F}_{(2,133)}=7.17, p=0.002\right)$, as well as a decrease in yolk sac $\left(3 \mathrm{dpf}-\mathrm{F}_{(2,136)}=11.96, p<0.001 ; 5 \mathrm{dpf}-\mathrm{F}_{(2,134)}=16.59\right.$, $p<0.001)$. Cardiac rate was further affected by noise temporal variation at $3 \mathrm{dpf}\left(\mathrm{F}_{(4,146)}=25.36, p<0.001\right)$ and $5 \mathrm{dpf}\left(\mathrm{F}_{(4,143)}=15.50, p<0.001\right)$, as well as the yolk sac $3 \mathrm{dpf}\left(\mathrm{F}_{(4,145)}=13.79, p<0.001\right)$ and $5 \mathrm{dpf}\left(\mathrm{F}_{(4,143)}=12.19\right.$, $p<0.001)$. Error bars represent $95 \%$ confidence intervals. Different letters indicate statistically significant differences between specific groups based on post hoc tests. (E) Correlation between cardiac rate and yolk sac size $(\mathrm{R}=-0.61, \mathrm{~N}=370, p<0.001)$ from both 3 and $5 \mathrm{dpf}$. Solid line-best-fitted line; inner dashed linesstandard error of the mean; outer dashed lines-95\% confidence interval.

In the present study, the intermittent treatment with many onsets of acoustic disturbance (IN1, characterized by $5-12$ s noise and $1-120$ s silence, about $15 \%$ total noise) caused higher mortality up to $33 \%$, similar to continuous noise exposure and IN2 (with similar noise exposure to IN1 but extended silent periods of 1-10 



Figure 3. Whole-body cortisol levels from larval zebrafish exposed to (A) continuous noise at different amplitudes ( $\left.3 \mathrm{dpf}: \mathrm{F}_{(2,35)}=4.84, p=0.014 ; 5 \mathrm{dpf}: \mathrm{F}_{(2,29)}=4.37, p=0.023\right)$ and $(\mathrm{B})$ varying noise temporal patterns at $150 \mathrm{~dB}$ re $1 \mu \mathrm{Pa}\left(3 \mathrm{dpf}: \mathrm{F}_{(4,45)}=2.23, p=0.082 ; 5 \mathrm{dpf:} \mathrm{F}_{(4,35)}=2.65, p=0.052\right)$. Control- silent conditions, $\mathrm{CN}$ continous noise at either $130\left(\mathrm{CN}_{130}\right)$ or $150 \mathrm{dBre} 1 \mu \mathrm{Pa}\left(\mathrm{CN}_{150}\right)$, IN- intermittent regime with short (IN1), medium (IN2) and long noise segments (IN3). Error bars represent 95\% confidence intervals. Different letters indicate statistically significant differences between specific groups based on post hoc tests.

min). This was significantly above baseline levels in control group (15\%) and contrasted with the IN3 treatment with prolonged noise presentations (total $50 \%$ noise presence) but fewer onsets that caused $27 \%$ in mortality. The relevance of the timing of acoustic exposure has received limited attention but increasing evidence points towards a significant impact on development and physiological stress in different fish species ${ }^{13,60}$. Nedelec et al. (2015) reported no difference in body length of larval codfish exposed to either regular or random playback of ship noise (average 15 min noise playback/hour) ${ }^{13}$. The body width-length ratio, however, declined over the course of the study and the greatest decline was registered under regular noise treatment at 16 days post-hatch. These larvae were easier to catch in a predator-avoidance experiment, demonstrating that the timing of acoustic disturbance can impact survival-related measures during development. Additionally, Neo et al. ${ }^{60}$ assessed the impact of continuous versus intermittent regular noise ( $0.1 \mathrm{~s}$ noise plus $0.9 \mathrm{~s}$ silence) on seabass adults (Dicentrarchus labrax, Moronidae) and found that intermittent exposure resulted in slower normal behaviour recovery compared to continuous treatment.

The present study also found heightened cardiac rate, yolk sac consumption, and cortisol levels at both 3 and $5 \mathrm{dpf}$ zebrafish treated with elevated noise levels, which is a clear indication of noise-induced physiological stress. The control cardiac rate values varied between 173 and 191 ( 3 and $5 \mathrm{dpf}$, respectively), which is comparable to prior studies on the same model organism ${ }^{61-63}$. We verified an increase of about $10 \%$ in both ontogenetic stages (3 dpf: from 173 to $191 \mathrm{bpm} ; 5 \mathrm{dpf}$ : 203-224 bpm) under increased noise conditions $\left(\mathrm{CN}_{150}\right)$. Simpson et al. ${ }^{48}$ and Jain-Schlaepfer et al. ${ }^{64}$ reported first evidences of higher cardiac activity due to anthropogenic noise in fish larvae. The authors found that the embryos (staghorn damselfish Amblyglyphidodon curacao and clownfishes Amphiprion spp.) increased their heart rate (up to 5\%) in the presence of increased levels of shipping noise, and that the physiological impact depended on the engine type.

Increased cardiac activity represents an adrenergic stress response, which is typically responsible for activating metabolic pathways and mobilizing energy to cope with potential challenges ${ }^{65-69}$. In this case, the perceived challenge consisted of acoustic disturbance, which is not a life-threatening situation. Hence, the energy depletion due to acoustic stress might be detrimental to the embryos that could otherwise use it for survival-related developmental processes. 


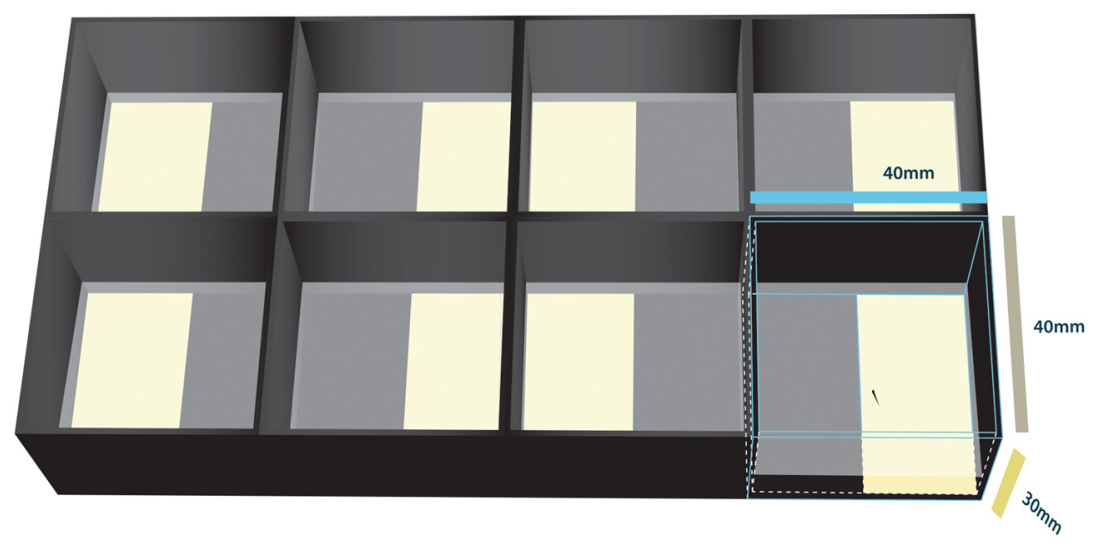

A

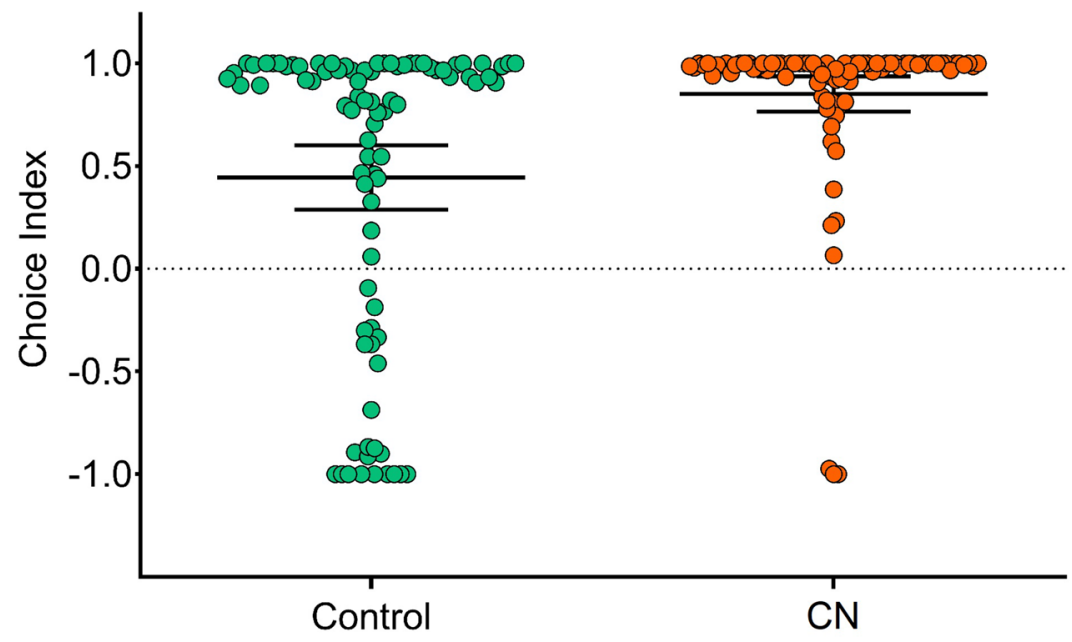

Figure 4. (A) Light/dark preference assay consisting of squared plastic compartments (each $40 \mathrm{~mm}$ width $\times 40 \mathrm{~mm}$ length $\times 30 \mathrm{~mm}$ height) divided into two equal sized areas with distinct bottom illumination (transparent/bright versus opaque/dark). The apparatus was placed on top of a LED panel ( 7000 lx). Each compartment was filled with $10 \mathrm{ml}$ water and a single larval zebrafish (5 dpf) was placed in the middle of the arena and recorded for $5 \mathrm{~min}$. (B) Choice index for larva exposed to continuous noise (150 dB re $1 \mu \mathrm{Pa})$ versus control conditions $\left(\mathrm{U}_{(2,173)}=5341.50, p<0.001\right)$. Choice index was calculated as: (Time in dark-Time in light)/(Time in dark + Time in light). Individual data are presented as scatter plots and bars depict mean $\pm 95 \%$ confidence intervals. Illustration was created using Adobe Illustrator CC 2020 version 24.0.2 for Windows, Adobe Inc., CA, USA and Blender version 2.80 for Windows, Blender Foundation, AMS, EU.

To our knowledge, the present study is the first to measure cardiac rate and related it with endogenous embryonic energy substrates (yolk sac size) in fish. We provide evidence that these variables are significantly correlated. In addition, we confirmed the heightened physiological stress in noise-exposed larvae by measuring their cortisol levels that were significantly above control groups. Chronic exposure to an environmental stressor may interfere with resource allocation from reserves maintenance to activation of the adrenal system, resulting in allostatic load ${ }^{70}$. Our study shows that larval zebrafish under noise exposure $\left(\mathrm{CN}_{150}\right)$ consume their yolk sac $21 \%$ (at $3 \mathrm{dpf}$ ) and $58 \%$ (at $5 \mathrm{dpf}$ ) faster compared to baseline conditions. Considering that noise-exposed individuals showed similar development (body size) to control group, we predict that the increased yolk consumption is not being invested in a faster development but reflects additional survival costs to cope with acoustic stress. The increased mortality registered in the noise-treated groups further supports this hypothesis. Nedelec et al. (2015) also reported an effect of shipping noise on yolk sac consumption in larval codfish ${ }^{13}$. Larvae exposed to regular noise used their yolk sac $29 \%$ faster after 2 days of exposure and had a lower body width-length ratio after 16 days post-hatch compared to specimens raised in quieter ambient noise conditions. Other studies have used the yolk sac size of larval fish to assess the impact of environmental stress ${ }^{71-74}$, and likewise showed that variables like salinity, temperature, light conditions and even maternal stress, can lead to a significant impact on the yolk sac absorption rate and composition.

Our work provides first evidence of noise-induced increase in whole-body cortisol levels in a larval fish. Cortisol elevation due to environmental stress, such as salinity, temperature, light conditions, acidity, mechanical disturbance, has been previously observed in larval zebrafish. While measuring units vary between studies, 

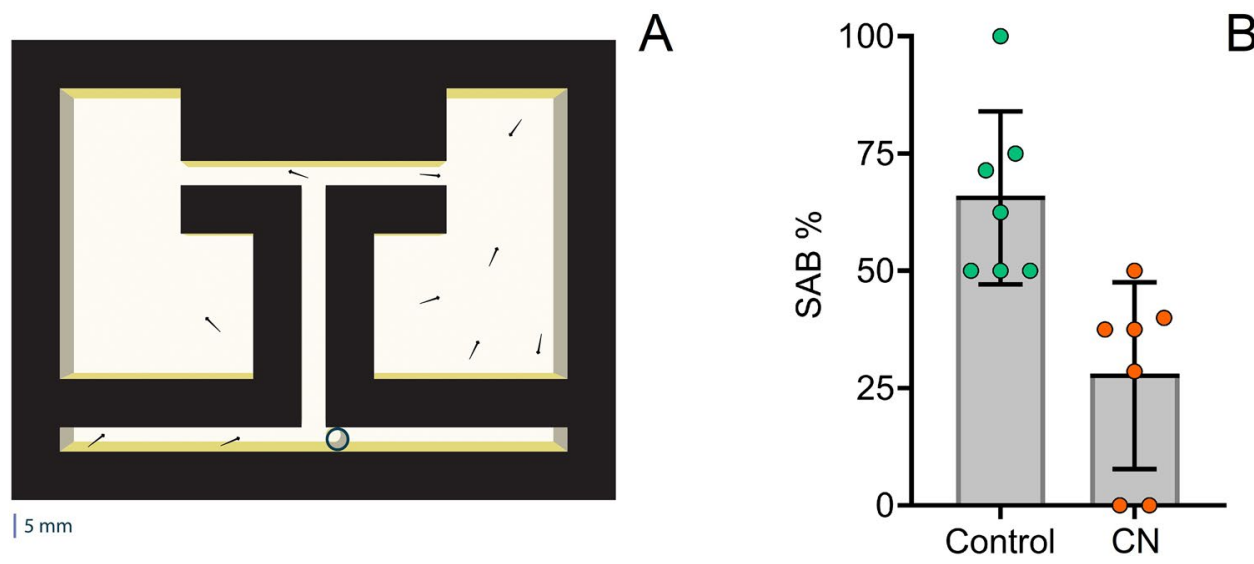

Figure 5. (A) Spontaneous Alternation Behaviour (SAB) assay with bottom illumination to test exploratory swimming and spatial memory. The starting arms can be used alternatively (a plastic tube blocks the entrance to the opposite arm) and converge into a perpendicular main arm that leads to a choice of alternation or same side arm. These arms lead to distinct pools of $19.50 \mathrm{~mm}^{2}$. (B) Comparison of SAB in $5 \mathrm{dpf}$ under continuous noise at $150 \mathrm{dBre} 1 \mu \mathrm{Pa}(\mathrm{CN})$ and control conditions $\left(\mathrm{t}_{(113)}=-4.08, p<0.001\right)$. From a total of 180 tested larvae, 115 successfully showed alternation behavior (entered the opposite side pool) within the 10-min recording. Individual data are presented as scatter plots and bars depict mean $\pm 95 \%$ confidence intervals. Illustration was created using Adobe Illustrator CC 2020 version 24.0.2 for Windows, Adobe Inc., CA, USA and Blender version 2.80 for Windows, Blender Foundation, AMS, EU.

cortisol levels are consistently within a range of 5-10 pg/larva for control and 200-250 pg/larva for stressed individuals (within 3-7 dpf) ${ }^{75-79}$. For instance, Bait et al..$^{78}$ reported an elevation in cortisol/protein of about $65 \%$ under cold conditions, $40 \%$ under UV light and almost $100 \%$ under mechanical disturbance. Our study reported a similar increase in cortisol levels of up to $95 \%$ ( $3 \mathrm{dpf}$ ) and $47 \%$ (5 dpf) due to acoustic stress.

Variation in the temporal patterns of intermittent treatments caused different effects on cardiac rate, yolk sac consumption, and nearly on cortisol levels (although not statistically significant), suggesting that time regime of the acoustic disturbance is important to down-regulate physiological stress. Overall, continuous noise exposure induced the highest cardiac rate and yolk consumption compared to intermittent treatments, whilst IN3 characterized by longer noise playback but also prolonged silent intervals caused less impact on these variables compared to control and other IN treatments. Moreover, cortisol levels were generally elevated in noise-exposed groups, and the highest level reached under continuous noise treatment. Although not significantly different, IN3 induced lower cortisol level compared to IN2. Similar to $\mathrm{Nedelec}^{13}$, we hypothesize that longer silent intervals during random disturbances allow for recovery, compensation and/or habituation in larval fish, and that the total duration of noise exposure is less crucial compared to the time regime adopted (number of onsets of acoustic disturbance and silent intervals).

Comparing the effects of noise between 3 and $5 \mathrm{dpf}$ larvae, we can conclude that the impact was generally more pronounced at the later developmental stage. The $5 \mathrm{dpf}$ larvae have remained chronically exposed to the acoustic treatments for a significantly longer period of time, which most likely resulted in higher cumulative effects. Although both stages are sensitive to sound, the inner ear saccule of $5 \mathrm{dpf}$ is more sensitive to auditory stimuli $^{80}$, and this may have contributed for higher acoustic stress. Future work should investigate the most sensitive window during embryonic development for acoustic stress, the consequences for the structure and function of auditory pathways, and associated metabolic costs.

The present study also investigated the impact of noise exposure $\left(\mathrm{CN}_{150}\right)$ at the behavioural level in larval zebrafish. We focused on $5 \mathrm{dpf}$, a developmental stage when larvae acquire full motility, display active feeding and danger/predator avoidance, suggesting that simple neural circuitries for processing reward and aversion are already functional ${ }^{81,82}$. We tested the effect of $\mathrm{CN}_{150}$ treatment on $5 \mathrm{dpf}$ larvae using the anxiety-related light/ dark preference test, which has been widely used to test stress and anxiety in mammals ${ }^{83}$ and zebrafish ${ }^{78,84-87}$. Our results indicate increased darkness aversion (scotophobia) in noise-exposed larvae, suggesting that such environmental stressor elicits anxiety behaviour. Similarly, Bai et al. ${ }^{78}$ reported that heat, cold and UV treatment significantly enhanced darkness aversion in larval zebrafish. The authors also found that treatment with two anxiolytics with different pharmacokinetics (chlordiazepoxide, a GABAergic benzodiazepine and buspirone, a serotonin agonist) attenuated this behavior, which confirmed that such pattern was anxiety-driven. Future research should consider testing the effect of different anxiolytics on noise-treated zebrafish and evaluate light/ dark preference at different development stages.

Finally, this study also showed that noise-exposed 5 dpf larvae $\left(\mathrm{CN}_{150}\right)$ displayed impaired innate Spontaneous Alternation Behaviour compared to control individuals. Bögli et al. ${ }^{88}$ effectively established the presence of SAB in larval zebrafish ( $6 \mathrm{dpf}$ ) suggesting the presence of early mnestic capabilities. At this developmental stage (4-5 dpf), the hippocampal-like pallium develops ${ }^{89}$, and this brain structure is known to be related with navigation and spatiotemporal sensing in fishes ${ }^{90,91}$. Other studies have investigated the effect of acoustic experience in memory function. For instance, Cheng et al. ${ }^{92}$ reported impaired learning and memory capabilities 
in mice after exposure to white noise at $80 \mathrm{~dB}$ SPL for $2 \mathrm{~h}$ per day for a 6-week period, along with peroxidative damage and tau hyperphosphorylation in different brain structures including the hippocampus. Other authors have reported that environmental noise may induce stress-like effects in juvenile rats affecting spatial learning abilities, and that stress in developing rats can affect behavioral lateralization ${ }^{93,94}$.

Additionally, the overall locomotor activity of these specimens was further investigated and a significant reduction in covered area was observed in the noise-treated larvae. These results are in line with Bhandiwad et al. ${ }^{54}$, who investigated the effect of noise exposure (white noise at $20 \mathrm{~dB}$ re $1 \mathrm{~ms}^{-2}$ ) on larval zebrafish (5-7 $\mathrm{dpf}$ ) and identified a significant decrease in total distance covered compared to control. Ongoing research aims to confirm whether impaired SAB resulted from spatial memory dysfunction and/or changes in the exploratory behaviour that are anxiety-driven or related to motor function. Additional work should evaluate the effect of different acoustic parameters (amplitude, temporal regime, spectral content) of noise exposure on behavioural patterns in larval zebrafish.

In summary, we provide first evidence of noise-induced physiological stress and behavioural disturbance in larval zebrafish, showing that increased noise amplitude and changes in the noise temporal regimes can induced higher cardiac rate and activate the adrenal system leading to increased cortisol levels and depletion of embryonic endogenous energy reserves. Intermittent sounds with short duration, such as those commonly found in aquatic systems with elevated traffic activity from small motor boats, speed boats and personal water crafts ${ }^{95-99}$, may have a stronger physiological and behavioural consequences on fish, including higher mortality, physiological stress and behavioural alterations, compared to regimes of similar or higher overall noise exposure but less number of noise onsets and longer silent periods.

Future studies are required to evaluate whether noise-induced energetic costs may result in carryover effects to subsequent life stages and fitness. We show that larval zebrafish can be established as a high-throughput platform for fast screening of the biological impact of acoustic disturbances at developmental, physiological and molecular levels. Furthermore, we highlight the importance of noise regularity and its consideration for noise management and mitigation strategies.

\section{Methods}

Fish husbandry and sampling. Zebrafish eggs were obtained from wild type adults (AB line) purchased from China Zebrafish Resource Center (CZRC, China) and reared at the research facilities of the University of Saint Joseph (Macao) following general guidelines for zebrafish housing and husbandry by Westerfield (2007) ${ }^{100}$. Stockfish were maintained in a standalone housing system (model AAB-074-AA-A, Yakos 65, Taiwan) with filtered and aerated water ( $\mathrm{pH}$ balanced $7-8 ; 400-550 \mu \mathrm{S}$ conductivity) at $28 \pm 1{ }^{\circ} \mathrm{C}$ and under a $12: 12$ light/dark cycle.

For each experimental trial, eggs were collected within $2 \mathrm{hpf}$ (hours post fertilization) from 2 to 6 breeding tanks (each tank containing about 10 females and 5 males). Collected eggs were mixed in a single Petri dish containing embryo medium and distributed into different groups of 50 specimens each. Depending on the experiment, either 3 or 5 groups were considered (see details below). This split-brood approach was adopted to minimize potential differences related to egg quality/viability. Each group was allocated to a different acoustic treatment tank that was randomly assigned for a specific treatment. The sample size ( 50 specimens per treatment group) was selected to ensure enough specimens for data collection in the multiple tests, and also based on previous observations of mortality rates.

Egg survivability was evaluated during the first $48 \mathrm{hpf}$ after examination of the batches at a fixed time in the morning (between 10 and $11 \mathrm{am}$ ). Morphological and physiological data was consistently collected at the same time in the morning at two developmental stages, 3 and $5 \mathrm{dpf}$. These stages of development were selected since at $3 \mathrm{dpf}$ embryos already have a functioning inner ear ${ }^{101}$, and at $5 \mathrm{dpf}$ specimens already exhibit active feeding and sensorimotor behaviors, such as auditory-evoked escape responses, that are affected by noise exposure ${ }^{54}$.

All specimens that were used in experimental procedures were euthanized at the end in $300 \mathrm{mg} / \mathrm{L}$ of Tricaine Methanesulfonate (MS-222, Thermo Fisher Scientific INC, Massachussets, USA) based on the protocol by Strykowski and Schech $(2015)^{102}$.

All experimental procedures complied with the ethical guidelines regarding animal research and welfare enforced at the Institute of Science and Environment, University of Saint Joseph, and approved by the Division of Animal Control and Inspection of the Civic and Municipal Affairs Bureau of Macao (IACM), license AL017/ DICV/SIS/2016.

Experimental design and acoustic treatments. A total of 15 experimental trials consisting of simultaneous treatments of either different noise amplitudes or varying temporal patterns (at the same noise level each) were conducted. The acoustic treatments were carried in glass tanks $(60 \mathrm{~cm}$ length $\times 30 \mathrm{~cm}$ width $\times 50 \mathrm{~cm}$ height $)$ equipped with top built-in illumination ( $7000 \mathrm{Lux}$ in a 12:12 light/dark cycle) and covered with a Styrofoam structure to control for light, temperature and noise conditions. No filtering system was used to avoid additional noise, but complete water changes were carried between trials to maintain appropriate water quality similar to stock conditions. Each treatment tank was mounted on top of Styrofoam boards placed over two granite slabs $(1.5 \mathrm{~cm}$ thick) spaced by rubber pads to reduce non-controlled transmission of building vibrations. Eggs were placed inside a custom-made cylindrical fine-mesh net box $(5 \mathrm{~cm}$ diameter, $6 \mathrm{~cm}$ high) suspended at $\sim 7 \mathrm{~cm}$ above an underwater speaker (UW30, Lubel Labs, Ohio, USA) that rested on top of a sponge base to minimize transmission of playback vibrations into the tank bottom (Fig. 6A). Speakers were connected to audio amplifiers (ST-50, Ai Shang Ke, China) that were connected to laptops running Adobe Audition 3.0 for windows (Adobe Systems Inc., USA). A total of 5 experimental tanks were used alternately for the different treatments across the various trials. In "control" group, the amplifier was connected to the speaker and switched on, however there was 

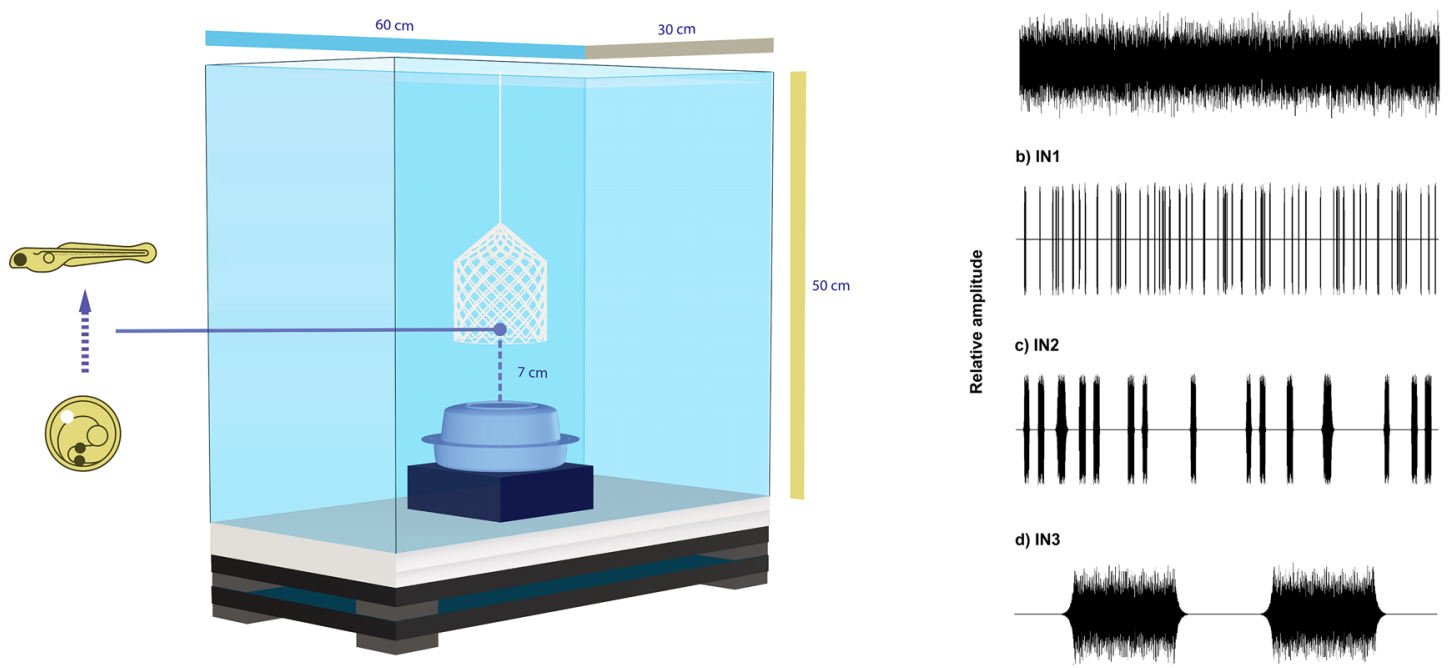

Figure 6. (A) Diagram of the acoustic treatment tank. The tank rested on top of two granite plaques separated by anti-vibratory rubber pads. Inside, a custom-made net cylinder containing zebrafish egg/larvae was suspended $7 \mathrm{~cm}$ above an underwater speaker (UW30, Lubel Labs, Ohio, USA) that rested on top of a polyurethane sponge. (B) Oscillogram of sound files used for playbacks. Control- silent conditions, CNcontinuous noise at either $130\left(\mathrm{CN}_{130}\right)$ or $150 \mathrm{~dB}$ re $1 \mu \mathrm{Pa}\left(\mathrm{CN}_{150}\right)$, IN- intermittent regime with random short noise segments (IN1): 5-12 s duration spaced by silent intervals of 1-120 s (total noise exposure of c. 15\%); medium noise segments (IN2): 30-60 s interspaced by 1-10 min silence (similar noise exposure to IN1); and long noise segments (IN3) of 15 min separated by 15 min silent periods (about $50 \%$ overall noise). Illustration was created using Adobe Illustrator CC 2020 version 24.0.2 for Windows, Adobe Inc., CA, USA and Blender version 2.80 for Windows, Blender Foundation, AMS, EU.

no playback. The background noise level measured in such control condition varied between 103 and $108 \mathrm{~dB}$ re. $1 \mu \mathrm{Pa}$ (LZS, RMS sound level obtained with slow time and linear frequency weightings: $6.3 \mathrm{~Hz}-20 \mathrm{kHz}$ ).

Sound treatments consisted of white noise low-pass filtered at $1500 \mathrm{~Hz}$ and adjusted to the tank acoustic properties using Adobe Audition software tools to deliver a relative flat spectrum. To test for the effects of amplitude on larval zebrafish, two sound files of continuous white noise $(\mathrm{CN})$ at different amplitudes or Sound Pressure Levels (SPLs), namely $130 \mathrm{~dB}\left(\mathrm{CN}_{130}\right)$ and $150 \mathrm{~dB}$ re. $1 \mu \mathrm{Pa}\left(\mathrm{CN}_{150}\right)$, were generated and played back in loop. These noise levels were similar to those found in freshwater aquatic habitats characterized by anthropogenic noise activity such as shipping ${ }^{103,104}$ and noise conditions in certain zebrafish housing systems ${ }^{55}$. To evaluate the impact of different noise temporal regimes, three additional sound files of intermittent noise and 60 min duration were generated and played back in loop at $150 \mathrm{~dB}$ re $1 \mu \mathrm{Pa}$ (Fig. 6B): IN1- short noise segments of 5-12 s duration spaced by silent intervals of 1-120 s (60 noise events per hour) reaching an overall noise exposure of about $15 \%$, designed to mimic intense boat traffic noise as described in Nichols et al. $(2015)^{95}$; IN2- medium noise segments of 30-60 s interspaced by 1-10 min silence, with 15 noise segments per hour and similar overall noise exposure to IN1; and IN3- long noise segments of $15 \mathrm{~min}$ separated by $15 \mathrm{~min}$ silent periods (about $50 \%$ overall noise), which followed a prolonged shipping activity as described by Nedelec et al. (2015) ${ }^{13}$ (Fig. 6B). All noise presentations contained a logarithmic fade-in and fade-out ramps of $10 \%$ of the noise presentation. We used random exposure regimes since it reflects better an acoustic environment characterized by traffic noise and it is known to cause physiological stress in fish ${ }^{13,95}$, and affect specifically zebrafish behaviour ${ }^{105}$.

Noise levels were calibrated before each experimental trial so that the intended sound level (LZS, $6.3 \mathrm{~Hz}-20 \mathrm{kHz})$ was reached at the bottom of the net box $(\sim 7 \mathrm{~cm}$ above the speaker $)$ using a hydrophone (Bruel\&Kjær 8104, Naerum, Denmark; frequency range: $0.1 \mathrm{~Hz}$ to $120 \mathrm{kHz}$, sensitivity of - $205 \mathrm{~dB} \mathrm{re} 1 \mathrm{~V} / \mu \mathrm{Pa}$ ) connected to a hand-held sound level meter (Bruel\&Kjær model 2270). Additionally, the acoustic treatments were calibrated with a tri-axial accelerometer (M20-040, frequency range: $1-3 \mathrm{kHz}$, GeoSpectrum Technologies, Dartmouth, Canada). The particle acceleration sensor was placed horizontally with the acoustic center also positioned at about $7 \mathrm{~cm}$ from the speaker in the position occupied by the net box containing the specimens. Particle acceleration level determined for the highest sound level $(150 \mathrm{~dB}$ re $1 \mu \mathrm{Pa})$ in the vertical axis consisted of $120 \mathrm{~dB} / \mathrm{s}^{2}$. A decrease of $20 \mathrm{~dB}$ in sound pressure resulted in similar variation in acceleration. The sound playbacks generated most energy in the vertical axis compared to the other orthogonal directions. The calibration was conducted using a Matlab script (paPAM) based on Nedelec et al. (2016)

Morphological and cardiac rate measurements. The impact of the acoustic treatments on morphological development and cardiac rate of larval zebrafish was assessed based on 5 specimens per treatment in each experimental trial. The specimens were lightly sedated using low concentration $(0.004 \%)$ of MS-222 buffered with sodium bicarbonate to quantify cardiac activity following previously described procedures ${ }^{107}$, and then observed under a stereomicroscope (Stemi 2000CS, Carl Zeiss, Jena, Germany) connected to a digital camera 
(Axiocam, ICc3, Carl Zeiss) and a desktop running Zen 2.3 Lite (Carl Zeiss). Photographs were taken from each specimen in lateral position for determining total body length and yolk sac size based on Teixidó et al. ${ }^{108}$, followed by a video of $60 \mathrm{~s}$ focusing on the cardiac area.

Both photographs and videos were analysed using specific tools for morphological analysis and automatic detection of cardiac activity of DanioScope software (Noldus Information Technology, Wageningen, Netherlands). The yolk sac size was defined as the lateral area (xy coordinates) delineated manually. The cardiac rate measurement was also done manually to validate the software automatic measurements and whenever the video quality was not appropriate.

The experimenter remained unaware of the treatment of the specimens under analysis (blinded experiment), since files were automatically labelled by the software with an alphanumeric code following the sequence of data acquisition. The information regarding the treatment of the specific file number was registered in a document that was subsequently used to organize information into a data sheet.

Cortisol quantification. The analyses were conducted based on 10 larvae collected as one sample from the different treatments and for the two developmental stages analyzed. A total of eight different trials were carried to test the effect of increasing noise level, and six trials to assess the impact of different noise temporal patterns. We followed previously described procedures by Bai et al. ${ }^{78}$ adapted to improve extraction process. Specimens were euthanized in MS-222 $300 \mathrm{mg} / \mathrm{L}$, excess water was removed, and individuals frozen at $-80{ }^{\circ} \mathrm{C}$ in collection tubes. Prior to analysis, samples were thawed by adding $150 \mu \mathrm{l}$ of ice-cold Millipore Ultrapure water to each tube and homogenized with a hand-held pellet mixer for $20 \mathrm{~s}$. Immediately, $450 \mu \mathrm{l}$ of pure methanol were added to each sample and vortexed at max speed for $30 \mathrm{~s}$. Samples were placed at $4{ }^{\circ} \mathrm{C}$ for $1 \mathrm{~h}$, vortexed again, and centrifuged at $3000 \mathrm{~g}$ for $10 \mathrm{~min}$ at $4{ }^{\circ} \mathrm{C}$. The liquid phase was carefully collected and transferred to a new vial. The methanol extraction was again repeated following same procedure and the liquid phase collected into the same vial. Liquid nitrogen-evaporated samples were reconstituted in $500 \mu \mathrm{l}$ of enzyme immunoassay buffer and kept at $-80^{\circ} \mathrm{C}$ for less than $24 \mathrm{~h}$, and vortexed prior to assay. Samples were tested in duplicate using a colorimetric cortisol enzyme immunoassay kit (Cayman Chemical, Ann Arbor, MI, USA).

Light/dark choice assay. Larval zebrafish ( $5 \mathrm{dpf})$ exposed to either continuous noise $\left(\mathrm{CN}_{150}\right)$ or control conditions were tested with the anxiety-related light/dark preference assay, which is a known behavioural paradigm to assess environmental stress in larval zebrafish ${ }^{78}$. Increased percentage of time spent in the light side is assumed to reflect increased anxiety in this model organism, which was previously found to prefer light or show dark avoidance (scotophobia) ${ }^{109}$. The light/dark preference apparatus was designed based of Bai et al. ${ }^{78}$ and consisted on eight individual squared transparent plastic compartments $(4.0 \mathrm{~cm}$ length $\times 4.0 \mathrm{~cm}$ width $\times 3.0 \mathrm{~cm}$ height), each divided into two equal sized areas with distinct bottom illumination conditions-transparent/ bright and opaque/dark (Fig. 4A). The apparatus was placed on top of a LED panel covered with a white translucent glass to provide uniform illumination $(\sim 7000 \mathrm{~lx})$. The sides of the compartments were covered with black vinyl tape to control for external visual interferences. Each compartment was filled with $10 \mathrm{ml}$ of system water at about $28^{\circ} \mathrm{C}$ (water depth: $\sim 5 \mathrm{~mm}$ ). A single larva was carefully placed with a plastic pipette in the middle region between the two areas in each compartment and a 5 min video covering the multiple testing compartments was recorded. A total of 11 trials with simultaneous test compartments were conducted. The videos were analysed regarding the time spent in each area and a Choice Index calculated: time spent in darkness-time spent in light $) /($ time spent in darkness + time spent in light). Meaning that $(0)$ reflects no preference, $(-1)$ larva spent all the time swimming in the light zone, and (1) all the time in the dark zone (Fig. 4B).

Spontaneous alternation behaviour assay. Larval zebrafish were further tested regarding the Spontaneous Alternation Behaviour (SAB), which describes the tendency of animals to alternate their turn direction in consecutive turns while navigating through the environment ${ }^{56,110}$. Each choice statistically depends on the previous one but, unlike other memory assessment paradigms, SAB does not require prior training or reinforcement. Moreover, the first occurrence of SAB is tightly correlated with the development of the hippocampus in several vertebrates $^{56,111,112}$, which further evidences its mnestic origin.

The SAB was assessed in a 3D printed T-maze designed based on Bögli and Huang ${ }^{113}$ and consisted of two starting arms converging into a main arm that bifurcated in the end into two goal arms, each leading to a goal pool (Fig. 5A). Starting and main arms had a length of $5.0 \mathrm{~cm}$, while goal arms measured $2.5 \mathrm{~cm}$. All arms had a width of $0.5 \mathrm{~cm}$ and depth of $1.0 \mathrm{~cm}$, and the goal arenas were $19.50 \mathrm{~cm}^{2}$. The maze was illuminated from below by placing it on top of a LED panel. For each of the 9 trials, 10 larvae were tested simultaneously. The maze was prefilled with fresh system water at $28^{\circ} \mathrm{C}$ (to a depth of about $0.7-0.75 \mathrm{~cm}$ ) and the intersections between the two starting arms and the main arm were both initially closed with plastic tubes. Testing larvae were carefully placed simultaneously using a plastic pipette in one of the starting arms and, after 5 min of acclimation, the respective tube was removed to start the trial. Successful entry was counted when a larva fully entered one of the goal pools within the $10 \mathrm{~min}$ of the recording. In the case of returning to the main arm and/or entering the second goal pool, only the first entry was counted. In order to evaluate potential changes in motor behaviour due to acoustic stress, another set of specimens subject to the same treatments/control (10-15 per trial) were analysed regarding the total swimming area in a petri dish $(8 \mathrm{~cm}$ diameter) based on video analysis ( $5 \mathrm{~min})$. The swimming area of each individual was determined using a grid $(5 \times 5 \mathrm{~mm})$ and based on the total number of squares covered.

Data analysis. Comparisons of hatching rate, total length, mortality, cardiac rate, yolk sac size and cortisol levels between groups exposed to different noise levels and temporal patterns variations were based on One-way 
ANOVA tests. These were followed by LSD multiple comparison post hoc tests to verify pairwise differences. The relationship between cardiac rate and yolk sac size was further assessed with a Pearson's correlation coefficient. Additionally, given that control and continuous noise $\left(\mathrm{CN}\right.$ or $\left.\mathrm{CN}_{150}\right)$ conditions were the same in both experiments (testing the effects of different noise levels and varying temporal patterns) and results were not statistically different, the data collected in both experiments were merged and used for testing both effects.

The potential effects of the batch quality/trial were assessed through mixed models containing "batch" as a random factor, and no interaction between these variables was detected.

Differences in light/dark preference were quantified based on a Choice Index and compared between treatment groups based on a two-tailed Man-Whitney $U$ test. Finally, the presence of spontaneous alternation behaviour and the total swimming area were compared between noise-exposed and control groups using 2-tailed t-tests.

The assumptions for parametric analyses were confirmed through the inspection of normal probability plots and by performing the Levene's test for homogeneity of variances. All statistical tests were performed using SPSS v26 (IBM Corp. Armonk, NY, US) and Statistica 10 for Windows (Dell Software, Inc., Round Rock, T, USA).

Illustrations were created using Adobe Illustrator CC 2020 version 24.0.2 for Windows, Adobe Inc., CA, USA and Blender version 2.80 for Windows, Blender Foundation, AMS, EU. Graphs were created using GraphPad Prism version 8.0.1 for Windows, GraphPad Software, CA, USA.

Received: 11 December 2020; Accepted: 26 February 2021

Published online: 23 March 2021

\section{References}

1. Miedema, H., Janssen, S., Rokho, K. \& Brown, L. Burden of disease from environmental noise: quantification of healthy life years lost in Europe (2011).

2. Peris, E. Environmental noise in Europe: 2020. Eur. Environ. Agency 1, 104 (2020).

3. Merchant, N. D. Underwater noise abatement: Economic factors and policy options. Environ. Sci. Policy 92, 116-123 (2019).

4. Babisch, W. et al. Auditory and non-auditory effects of noise on health. NIH Lancet 23, 1-7 (2014).

5. Recio, A., Linares, C., Banegas, J. R. \& Díaz, J. Road traffic noise effects on cardiovascular, respiratory, and metabolic health: an integrative model of biological mechanisms. Environ. Res. 146, 359-370 (2016).

6. Shannon, G. et al. A synthesis of two decades of research documenting the effects of noise on wildlife. Biol. Rev. 91, 982-1005 (2016).

7. Halfwerk, W. et al. Low-frequency songs lose their potency in noisy urban conditions. Proc. Natl. Acad. Sci. 108, 14549-14554 (2011).

8. Kight, C. R. \& Swaddle, J. P. How and why environmental noise impacts animals: an integrative, mechanistic review. Ecol. Lett. 14, 1052-1061 (2011).

9. Whitfield, A. K. \& Becker, A. Impacts of recreational motorboats on fishes: a review. Mar. Pollut. Bull. 83, 24-31 (2014).

10. Popper, A. N. N. \& Hastings, M. C. C. The effects of anthropogenic sources of sound on fishes. J. Fish Biol. 75, 455-489 (2009).

11. Bejder, L., Samuels, A., Whitehead, H., Finn, H. \& Allen, S. Impact assessment research: use and misuse of habituation, sensitisation and tolerance in describing wildlife responses to anthropogenic stimuli. Mar. Ecol. Prog. Ser. 395, 177-185 (2009).

12. Wale, M. A., Simpson, S. D. \& Radford, A. N. Size-dependent physiological responses of shore crabs to single and repeated playback of ship noise. Biol. Lett. 9, 20121194 (2013).

13. Nedelec, S. L., Simpson, S. D., Morley, E. L., Nedelec, B. \& Radford, A. N. Impacts of regular and random noise on the behaviour, growth and development of larval Atlantic cod (Gadus morhua). Proc. R. Soc. B 282, 20151943 (2015).

14. Johansson, K., Sigray, P., Backström, T. \& Magnhagen, C. Stress response and habituation to motorboat noise in two coastal fish species in the Bothnian Sea. In The Effects of Noise on Aquatic Life II 273-279 (2016).

15. Holmes, L. J., McWilliam, J., Ferrari, M. C. O. \& McCormick, M. I. Juvenile damselfish are affected but desensitize to small motor boat noise. J. Exp. Mar. Bio. Ecol. 494, 63-68 (2017).

16. Reid, S. G., Bernier, N. J. \& Perry, S. F. The adrenergic stress response in fish: Control of catecholamine storage and release. Comp. Biochem. Physiol. C. Pharmacol. Toxicol. Endocrinol. 120, 1-27 (1998).

17. Rajalakshmi, R., John, N. A. \& John, J. Review on noise pollution and its associated health hazards. Sch. J. Appl. Med. Sci. 4, 500-503 (2016).

18. Christie, K. W. \& Eberl, D. F. Noise-induced hearing loss: new animal models. Curr. Opin. Otolaryngol. Head Neck Surg. 22, 374-383 (2014).

19. Ortega, C. P. Effects of noise pollution on birds: A brief review of our knowledge. Source Ornithol. Monogr. Ornithol. Monogr. 74, 6-22 (2012).

20. Simmons, A. M. \& Narins, P. M. Effects of anthropogenic noise on amphibians and reptiles. In Springer Handbook of Auditory Research 179-208 (Springer, 2018).

21. de Soto, N. A. et al. Anthropogenic noise causes body malformations and delays development in marine larvae. Sci. Rep. 3, 2831 (2013).

22. Soto, N. A. de. Peer-reviewed studies on the effects of anthropogenic noise on marine invertebrates: from scallop larvae to giant squid. Eff. Noise Aquat. Life II 875, 273-279 (2016).

23. Brouček, J. Effect of noise on performance, stress, and behaviour of animals. Slovak J. Anim. Sci 47, 111-123 (2014).

24. Tennessen, J. B., Parks, S. E. \& Langkilde, T. Traffic noise causes physiological stress and impairs breeding migration behaviour in frogs. Conserv. Physiol. 2, 1-8 (2014).

25. Erbe, C., Dunlop, R. \& Dolman, S. Effects of noise on marine mammals. In Effects of Anthropogenic Noise on Animals 277-309 (Springer, 2018).

26. Kunc, H. P., McLaughlin, K. E. \& Schmidt, R. Aquatic noise pollution: implications for individuals, populations, and ecosystems. Proc. R. Soc. B 283, 20160839 (2016).

27. van der Sluijs, I. et al. Communication in troubled waters: responses of fish communication systems to changing environments. Evol. Ecol. 25, 623-640 (2011).

28. Cox, B. S., Dux, A. M., Quist, M. C. \& Guy, C. S. Use of a seismic air gun to reduce survival of nonnative lake trout embryos: a tool for conservation?. N. Am. J. Fish. Manag. 32, 292-298 (2012).

29. Wysocki, L. E. et al. Effects of aquaculture production noise on hearing, growth, and disease resistance of rainbow trout Oncorhynchus mykiss. Aquaculture 272, 687-697 (2007).

30. Debusschere, E. et al. Acoustic stress responses in juvenile sea bass Dicentrarchus labrax induced by offshore pile driving. Environ. Pollut. 208, 747-757 (2016). 
31. Filiciotto, F. et al. Impact of aquatic acoustic noise on oxidative status and some immune parameters in gilthead sea bream Sparus aurata (Linnaeus, 1758) juveniles. Aquac. Res. 48, 1895-1903 (2017).

32. Smith, M. E., Kane, A. S. \& Popper, A. N. Noise-induced stress response and hearing loss in goldfish (Carassius auratus). J. Exp. Biol. 207, 427-435 (2004).

33. Vasconcelos, R. O., Amorim, M. C. P. \& Ladich, F. Effects of ship noise on the detectability of communication signals in the Lusitanian toadfish. J. Exp. Biol. 210, 2104-2112 (2007).

34. Hasan, M. R., Crane, A. L., Ferrari, M. C. O. \& Chivers, D. P. A cross-modal effect of noise: the disappearance of the alarm reaction of a freshwater fish. Anim. Cogn. 21, 419-424 (2018).

35. Herbert-Read, J. E., Kremer, L., Bruintjes, R., Radford, A. N. \& Ioannou, C. C. Anthropogenic noise pollution from pile-driving disrupts the structure and dynamics of fish shoals. Proc. R. Soc. B Biol. Sci. 284, 1-9 (2017).

36. Francis, C. D. \& Barber, J. R. A framework for understanding noise impacts on wildlife: an urgent conservation priority. Front. Ecol. Environ. 11, 305-313 (2013).

37. Ladich, F. Acoustic communication and the evolution of hearing in fishes. Philos. Trans. R. Soc. B Biol. Sci. 355, 1285-1288 (2000).

38. Radford, A. N., Kerridge, E. \& Simpson, S. D. Acoustic communication in a noisy world: can fish compete with anthropogenic noise?. Behav. Ecol. 25, 1022-1030 (2014).

39. Dooling, R. J. \& Popper, A. N. The effects of highway noise on birds. Environ. Bioacoust. 27, 1-74 (2007).

40. Blom, E. L. et al. Continuous but not intermittent noise has a negative impact on mating success in a marine fish with paternal care. Sci. Rep. 9, 1-9 (2019).

41. Bureš, Z., Popelár, J. \& Syka, J. Noise exposure during early development impairs the processing of sound intensity in adult rats. Hear. Res. 352, 1-11 (2017).

42. Dorado-Correa, A. M., Zollinger, S. A., Heidinger, B. \& Brumm, H. Timing matters: traffic noise accelerates telomere loss rate differently across developmental stages. Front. Zool. 15, 1-8 (2018).

43. Mueller, C. A. Critical Windows in Animal Development: Interactions Between Environment, Phenotype, and Time (Springer, 2018).

44. Gordon, T. A. C. et al. Acoustic enrichment can enhance fish community development on degraded coral reef habitat. Nat. Commun. 10, 1-7 (2019).

45. Radford, A. N., Lebre, L., Lecaillon, G., Nedelec, S. L. \& Simpson, S. D. Repeated exposure reduces the response to impulsive noise in European seabass. Glob. Change Biol. 22, 3349-3360 (2016).

46. Banner, A. \& Hyatt, M. Effects of noise on eggs and larvae of two estuarine fishes. Trans. Am. Fish. Soc. 102, 142-144 (1973).

47. Fakan, E. P. \& McCormick, M. I. Boat noise affects the early life history of two damselfishes. Mar. Pollut. Bull. 141, 493-500 (2019).

48. Jain-Schlaepfer, S., Fakan, E., Rummer, J. L., Simpson, S. D. \& McCormick, M. I. Impact of motorboats on fish embryos depends on engine type. Conserv. Physiol. 6, 1-9 (2018).

49. Brittijn, S. A. et al. Zebrafish development and regeneration: new tools for biomedical research. Int. J. Dev. Biol. 53, 835-850 (2009).

50. Magyary, I. Recent advances and future trends in zebrafish bioassays for aquatic ecotoxicology. Ecocycles 4, 12-18 (2018)

51. Sarmah, S. \& Marrs, J. A. Zebrafish as a vertebrate model system to evaluate effects of environmental toxicants on cardiac development and function. Int. J. Mol. Sci. 17, 1-16 (2016).

52. Varshney, G. K., Pei, W. \& Burgess, S. M. Using zebrafish to study human deafness and hearing regeneration. Monogr. Hum. Genet. 20, 110-131 (2016).

53. Uribe, P. M. et al. Larval zebrafish lateral line as a model for acoustic trauma. Eneuro 5, 0206-0218 (2018),

54. Bhandiwad, A. A., Raible, D. W., Rubel, E. W. \& Sisneros, J. A. Noise-Induced hypersensitization of the acoustic startle response in larval zebrafish. JARO 19, 741-752 (2018).

55. Lara, R. A. \& Vasconcelos, R. O. Characterization of the natural soundscape of zebrafish and comparison with the captive noise conditions. Zebrafish 8, 1-13 (2018).

56. Lalonde, R. The neurobiological basis of spontaneous alternation. Neurosci. Biobehav. Rev. 26, 91-104 (2002).

57. Bogli, S. Y. \& Huang, M. Y. Y. Spontaneous alternation behavior in larval zebrafish. J. Exp. Biol. 220, 171-173 (2017).

58. Bruintjes, R. \& Radford, A. N. Chronic playback of boat noise does not impact hatching success or post-hatching larval growth and survival in a cichlid fish. PeerJ 2, e594 (2014).

59. Davidson, J., Bebak, J. \& Mazik, P. The effects of aquaculture production noise on the growth, condition factor, feed conversion, and survival of rainbow trout Oncorhynchus mykiss. Aquaculture 288, 337-343 (2009).

60. Neo, Y. Y. et al. Temporal structure of sound affects behavioural recovery from noise impact in European seabass. Biol. Conserv. 178, 65-73 (2014)

61. Craig, M. P., Gilday, S. D. \& Hove, J. R. Dose-dependent effects of chemical immobilization on the heart rate of embryonic zebrafish. Lab Anim. (NY) 35, 40-47 (2006).

62. Barrionuevo, W. R. \& Burggren, W. W. O2 consumption and heart rate in developing zebrafish (Danio rerio): influence of temperature and ambient $\mathrm{O}_{2}$. Am. Physiol. Soc. 276, 505-513 (2013).

63. De Luca, E. et al. ZebraBeat: a flexible platform for the analysis of the cardiac rate in zebrafish embryos. Sci. Rep. 4, 1-13 (2014).

64. Simpson, S. D., Yan, H. Y., Wittenrich, M. L. \& Meekan, M. G. Response of embryonic coral reef fishes (Pomacentridae: Amphiprion spp.) to noise. Mar. Ecol. Prog. Ser. 287, 201-208 (2005).

65. Anderson, W. G. et al. Remote monitoring of heart rate as a measure of recovery in angled Atlantic salmon, Salmo salar (L.). Hydrobiologia 371-372, 233-240 (1998).

66. Armstrong, J. D. Heart rate as an indicator of activity, metabolic rate, food intake and digestion in pike Esox lucius. J. Fish Biol. 29, 207-221 (1986).

67. Svendsen, E. et al. Heart rate and swimming activity as stress indicators for Atlantic salmon (Salmo salar). Aquaculture 531, $735804(2020)$

68. Burleson, M. L. \& Silva, P. E. Cross tolerance to environmental stressors: effects of hypoxic acclimation on cardiovascular responses of channel catfish (Ictalurus punctatus) to a thermal challenge. Bone 23, 1-7 (2008).

69. Brown, C., Gardner, C. \& Braithwaite, V. A. Differential stress responses in fish from areas of high- and low-predation pressure. J. Comp. Physiol. B Biochem. Syst. Environ. Physiol. 175, 305-312 (2005).

70. McEwen, B. S. \& Stellar, E. Stress and individual. Arch Intern. Med. 153, 2093-2101 (1993).

71. Mccormick, M. I. Behaviorally induced maternal stress in a fish influences progeny quality by a hormonal mechanism. Ecology 79, 1873-1883 (1998).

72. Yabu, T., Ishibashi, Y. \& Yamashita, M. Stress-induced apoptosis in larval embryos of Japanese flounder. Fish. Sci. 69, 1218-1223 (2003).

73. Werner, I., Linares-Casenave, J., Van Eenennaam, J. P. \& Doroshov, S. I. The effect of temperature stress on development and heat-shock protein expression in larval green sturgeon (Acipenser mirostris). Environ. Biol. Fishes 79, 191-200 (2007).

74. Shi, Z. et al. Salinity stress on embryos and early larval stages of the pomfret Pampus punctatissimus. Aquaculture 275, 306-310 (2008). 
75. Wilson, K. S. et al. Physiological roles of glucocorticoids during early embryonic development of the zebrafish (Danio rerio). J. Physiol. 591, 6209-6220 (2013).

76. Tudorache, C., Ter Braake, A., Tromp, M., Slabbekoorn, H. \& Schaaf, M. J. M. Behavioral and physiological indicators of stress coping styles in larval zebrafish. Stress 3890, 121-128 (2015).

77. Alsop, D. \& Vijayan, M. M. Development of the corticosteroid stress axis and receptor expression in zebrafish. Am. J. Physiol. Regul. Integr. Comp. Physiol. 294, 711-719 (2008).

78. Bai, Y., Liu, H., Huang, B., Wagle, M. \& Guo, S. Identification of environmental stressors and validation of light preference as a measure of anxiety in larval zebrafish. BMC Neurosci. 17, 1-12 (2016).

79. Barton, B. A. \& Zitzow, R. E. Physiological responses of juvenile walleyes to handling stress with recovery in saline water. Progress. Fish-Cult. 57, 267-276 (1995).

80. Yao, Q., DeSmidt, A. A., Tekin, M., Liu, X. \& Lu, Z. Hearing assessment in zebrafish during the first week postfertilization. Zebrafish 13, 79-86 (2016).

81. Colwill, R. M. \& Creton, R. Imaging escape and avoidance behavior in zebrafish larvae. Rev. Neurosci. 22, 63-73 (2011).

82. Voellmy, I. K. et al. Acoustic noise reduces foraging success in two sympatric fish species via different mechanisms. Anim. Behav. 89, 191-198 (2014).

83. Belzung, C. \& Griebel, G. Measuring normal and pathological anxiety-like behaviour in mice: a review. Behav. Brain Res. 125, 141-149 (2001).

84. Sireeni, J. et al. Profound effects of glucocorticoid resistance on anxiety-related behavior in zebrafish adults but not in larvae. Gen. Comp. Endocrinol. 292, 130-138 (2020).

85. Basnet, R. M., Zizioli, D., Taweedet, S., Finazzi, D. \& Memo, M. Zebrafish larvae as a behavioral model in neuropharmacology. Biomedicines 7, 1-16 (2019).

86. Peng, X. et al. Anxiety-related behavioral responses of pentylenetetrazole-treated zebrafish larvae to light-dark transitions. Pharmacol. Biochem. Behav. 145, 55-65 (2016).

87. Stewart, A. et al. Neurophenotyping of adult zebrafish using the light/dark box paradigm. NeuroMethods 51, 157-167 (2011).

88. Bögli, S. Y. \& Huang, M.Y.-Y. Spontaneous alternation behavior in larval zebrafish. J. Exp. Biol. 220, 171-173 (2017).

89. Mueller, T., Dong, Z., Berberoglu, M. A. \& Guo, S. The dorsal pallium in zebrafish, Danio rerio (Cyprinidae, Teleostei). Brain Res. 1381, 95-105 (2011).

90. Fotowat, H., Lee, C., Jun, J. J. \& Maler, L. Neural activity in a hippocampus-like region of the teleost pallium are associated with navigation and active sensing. bioRxiv 8, 1-25 (2018).

91. Broglio, C. et al. Hallmarks of a common forebrain vertebrate plan: specialized pallial areas for spatial, temporal and emotional memory in actinopterygian fish. Brain Res. Bull. 66, 277-281 (2005).

92. Cheng, L., Wang, S. H., Chen, Q. C. \& Liao, X. M. Moderate noise induced cognition impairment of mice and its underlying mechanisms. Physiol. Behav. 104, 981-988 (2011).

93. Jauregui-huerta, F., Garcia-estrada, J. \& Gonzalez-perez, O. Early exposure to noise followed by predator stress in adulthood impairs the rat'sre-learning flexibility in Radial Arm Water Maze. Neuro Endocrinol. Lett. 31, 1-12 (2010).

94. Rodriguez, M. \& Afonso, D. Ontogeny of T-maze behavioral lateralization in rats. Physiol. Behav. 54, 91-94 (1993).

95. Nichols, T. A., Anderson, T. W. \& Ana, Š. Intermittent noise induces physiological stress in a coastal marine fish. PLoS ONE 10, $1-13(2015)$.

96. Neo, Y. Y. et al. Sound exposure changes European seabass behaviour in a large outdoor floating pen: effects of temporal structure and a ramp-up procedure. Environ. Pollut. 214, 26-34 (2016).

97. Celi, M. et al. Vessel noise pollution as a human threat to fish: assessment of the stress response in gilthead sea bream (Sparus aurata, Linnaeus 1758). Fish Physiol. Biochem. 42, 631-641 (2016).

98. Erbe, C. Underwater noise of small personal watercraft (jet skis). J. Acoust. Soc. Am. 133, EL326-EL330 (2013).

99. Department of the Environment and Water Resources. Comparative Assessment of the Environmental Performance of Small Engines Marine Outboards and Personal Watercraft. Environmental Science and Technology (2007).

100. Westerfield, M. The Zebrafish Book. A Guide for the Laboratory Use of Zebrafish (Danio rerio) 5th edn. (University of Oregon Press, Eugene, 2000).

101. Lu, Z. \& Desmidt, A. A. Early development of hearing in zebrafish. JARO 14, 509-521 (2013).

102. Strykowski, J. L. \& Schech, J. M. Effectiveness of recommended euthanasia methods in larval zebrafish (Danio rerio). J. Am. Assoc. Lab. Anim. Sci. 54, 81-84 (2015).

103. Amoser, S., Wysocki, L. E. \& Ladich, F. Noise emission during the first powerboat race in an Alpine lake and potential impact on fish communities. J. Acoust. Soc. Am. 116, 3789-3797 (2004).

104. Codarin, A., Wysocki, L. E., Ladich, F. \& Picciulin, M. Effects of ambient and boat noise on hearing and communication in three fish species living in a marine protected area (Miramare, Italy). Mar. Pollut. Bull. 58, 1880-1887 (2009).

105. Shafiei Sabet, S., Neo, Y. Y. \& Slabbekoorn, H. The effect of temporal variation in sound exposure on swimming and foraging behaviour of captive zebrafish. Anim. Behav. 107, 49-60 (2015).

106. Nedelec, S. L. et al. Particle motion: the missing link in underwater acoustic ecology. Methods Ecol. Evol. 7, 836-842 (2016).

107. Chan, P. K., Lin, C. C. \& Cheng, S. H. Noninvasive technique for measurement of heartbeat regularity in zebrafish (Danio rerio) embryos. BMC Biotechnol. 9, 1-10 (2009).

108. Teixidó, E. et al. Automated morphological feature assessment for zebrafish embryo developmental toxicity screens. Toxicol. Sci. 167, 438-449 (2019).

109. Lau, B. Y. B., Mathur, P., Gould, G. G. \& Guo, S. Identification of a brain center whose activity discriminates a choice behavior in zebrafish. Proc. Natl. Acad. Sci. U. S. A. 108, 2581-2586 (2011).

110. Bögli, S. Y., Huang, M.Y.-Y., Bogli, S. Y. \& Huang, M.Y.-Y. Spontaneous alternation behavior in larval zebrafish. J. Exp. Biol. 220, $171-173(2017)$

111. Frederickson, C. J. \& Frederickson, M. H. Developmental changes in open-field behavior in the kitten. Dev. Psychobiol. 12, 623-628 (1979).

112. Vecera, S. P., Rothbart, M. K. \& Posner, M. I. Development of spontaneous alternation in infancy. J. Cogn. Neurosci. 3, 351-354 (1991).

113. Bogli, S. Y. \& Huang, M.Y.-Y. Spontaneous alternation behavior in larval zebrafish. J. Exp. Biol. 220, 171-173 (2017).

114. Du Sert, N. P. et al. The arrive guidelines 2.0: updated guidelines for reporting animal research. PLoS Biol. 18, 1-12 (2020).

\section{Acknowledgements}

We are grateful to I. H. Lau and D. Gonçalves (University of Saint Joseph, Macao) for support with the experimental work and insights on cortisol quantification, respectively. We are thankful to M. Vieira and P. J. Fonseca (cE3c, Faculdade de Ciências, Universidade de Lisboa, Portugal) for advising on the accelerometer calibration. We thank João Tiago Tavares for the setup illustrations. This study was supported by the Science and Technology Development Fund (FDCT), Macao (ref. 036/2015/A1 and 046/2018/A2). 


\section{Author contributions}

R.V. conceived the idea and conceptualized the research. Both authors contributed to the design of the methodology. R.L. assembled and carried out the experiments, conducted data acquisition and analysis. Both authors contributed critically to the interpretation the results and wrote the manuscript. The study was conducted in compliance with ARRIVE guidelines ${ }^{114}$.

\section{Competing interests}

The authors declare no competing interests.

\section{Additional information}

Correspondence and requests for materials should be addressed to R.A.L. or R.O.V.

Reprints and permissions information is available at www.nature.com/reprints.

Publisher's note Springer Nature remains neutral with regard to jurisdictional claims in published maps and institutional affiliations.

(c) (i) Open Access This article is licensed under a Creative Commons Attribution 4.0 International License, which permits use, sharing, adaptation, distribution and reproduction in any medium or format, as long as you give appropriate credit to the original author(s) and the source, provide a link to the Creative Commons licence, and indicate if changes were made. The images or other third party material in this article are included in the article's Creative Commons licence, unless indicated otherwise in a credit line to the material. If material is not included in the article's Creative Commons licence and your intended use is not permitted by statutory regulation or exceeds the permitted use, you will need to obtain permission directly from the copyright holder. To view a copy of this licence, visit http://creativecommons.org/licenses/by/4.0/.

(C) The Author(s) 2021 